\title{
Optimization of Three Morphologic Algorithms for Arrhythmia Discrimination in Implantable Cardioverter Defibrillators
}

\author{
A Cebrian ${ }^{1}, \mathrm{~J}^{\text {Rey }}{ }^{1}, \mathrm{~J} \mathrm{Millet}^{1}, \mathrm{~F}_{\text {Castells }}{ }^{1}, \mathrm{R}_{\text {Garcia-Civera }}{ }^{2}$ \\ ${ }^{1}$ Department of Electronics Engineering, Polytechnic University of Valencia, Spain \\ ${ }^{2}$ Electrophysiology Laboratory, University Clinic Hospital of Valencia, Spain
}

\begin{abstract}
Discrimination between ventricular tachycardia (VT) and supraventricular tachycardia (SVT) in implantable cardioverter defibrillators (ICDs) is still an unsolved task due to the low specificity of traditional techniques based in rate, stability and onset. Several morphological published algorithms enhance VT vs. SVT discrimination by increasing algorithm complexity.

Three morphological published algorithms with increasing complexity have been selected: time domain (complex peak area comparison), simplified Wavelet and frequency domain (Fourier complex power spectra analysis and neural network) algorithms. All them have been reconstructed from published information and programmed in MATLAB. The algorithms has been optimized in order to obtain an improved classification and to work in a 16-bit microcontroller platform (Texas Instruments MSP430 microcontroller). A final test of the optimized algorithms has been accomplished using a classified unipolar and bipolar electrogram (EGM) database. The configurable parameters of the algorithms have been adjusted in order to maximize sensitivity (SE), specificity (SP) and accuracy (AC).
\end{abstract}

\section{Introduction}

The classic criteria used in Implantable Cardioverter Devices (ICDs) based on rate, stability and sudden onset calculations over electrogram (EGM), offer high sensitivity performance for arrhythmia detection. The requirements of these combined criteria in terms of computational cost are very low.

Despite the high sensitivity obtained using these combined criteria, specificity decreases in difficult cases, especially when discriminating between Ventricular Tachycardia (VT) and Supraventricular Tachycardia (SVT) [1].

An additional step based on morphological criteria can be employed to improve specificity, while maintaining sensitivity performance. In fact, several morphological published algorithms enhance VT vs. SVT discrimination, with the overcome of increasing algorithm complexity [2-5].

Three morphological algorithms with increasing complexity have been selected: time domain [2] (complex peak area comparison), simplified Wavelet [3-4] and frequency domain [5] (Fourier complex power spectra analysis and neural network) algorithms. The algorithms had been previously analyzed and compared in terms of computational cost [6].

In this study, the algorithms are evaluated in terms of performance with a reconstructed and optimized implementation in MATLAB and tested over a own EGM database. Comparison between algorithms has been carried out by computing sensitivity (SE), specificity (SP) and accuracy (AC).

\section{Algorithm implementations}

The selected morphological discrimination algorithms with increasing complexity have been reconstructed following author published information. All these algorithms were proposed to discriminate between VT vs SVT. Time domain was suggested for bipolar recordings, whereas the other two algorithms were conceived for unipolar EGMs. In this section it is reproduced a short description of each method.

\section{TIME DOMAIN}

This algorithm analyses the ventricular complex morphology on a beat-to-beat basis. Each complex is compared to a previously stored complex template from the baseline rhythm. The comparison is performed by computing the difference between the peak areas of the test and template complexes. Both complexes are aligned and the area of each peak is normalized (figure 1).

The algorithm implementation steps are as follows:

1. Obtain a baseline rhythm complex template, by averaging four consecutive complexes.

2. Extraction of one complex from the rhythm under analysis.

3. Alignment of both complexes.

4. Normalized complex peak area calculation.

5. Differences between peak areas are accumulated: 


$$
\text { area_dif }=\mid \text { area of } \mathrm{A}-\text { area of } \mathrm{A}^{\prime} \mid+
$$

$\mid$ area of $\mathrm{B}$ - area of $\mathrm{B}^{\prime}|+|$ area of $\mathrm{C}-$ area of $\mathrm{C}^{\prime} \mid$

6. Percent match score calculation:

$$
\operatorname{Match}(\%)=\frac{1}{\text { area_dif }} \cdot 100
$$

7. If the percent match score is greater than a programmable threshold (range $30 \%$ to $95 \%$ ) for a number of programmable complexes (typically 5 of 8 ), the discrimination algorithm indicates SVT, otherwise is classified as VT.

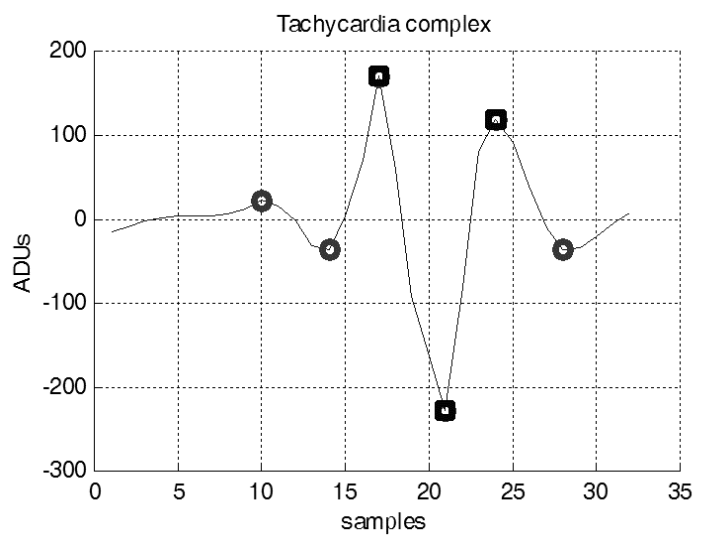

Figure 1. Complex peaks detection previous to peak area computation in time domain algorithm

\section{SIMPLIFIED WAVELET}

One of the proposed methodologies to discriminate VT vs. SVT is derived from the Wavelet transform. The algorithm is based in the fact that alteration on the morphology of the complexes is generally caused by a change in the origin chamber.

The algorithm compares the morphology of ventricular EGMs during a tachycardia with a template of electrogram morphology recorded during baseline rhythm using the Wavelet transform. A percent match score describes the degree of morphology similarity of the baseline and tachycardia EGMs (figure 2).

The algorithm implementation steps are as follows:

1. Obtain a baseline rhythm complex template, by averaging four consecutive complexes.

2. Extraction of one complex from the rhythm under analysis.

3. Alignment of both complexes.

4. Complexes simplified Wavelet transformation computation.

5. Wavelet coefficients filtering (coefficients smaller in absolute value than a programmed threshold are removed).

6. Wavelet filtered coefficients normalization.
7. Percent match score calculation.

8. If the percent match score is below than $70 \%$ for a number of programmable complexes (at least 6 of 8), the tachycardia is classified as ventricular in origin (VT). Otherwise, the tachycardia is classified as atrial in origin (SVT).

The simplified Wavelet transformation is based on the Haar Wavelet transform. The original Haar Wavelet transform has been modified defining the amplitude of all Wavelet functions to be either 1 or -1 . Using these amplitude values no products are needed, reducing hence the computational cost of the Wavelet transform.

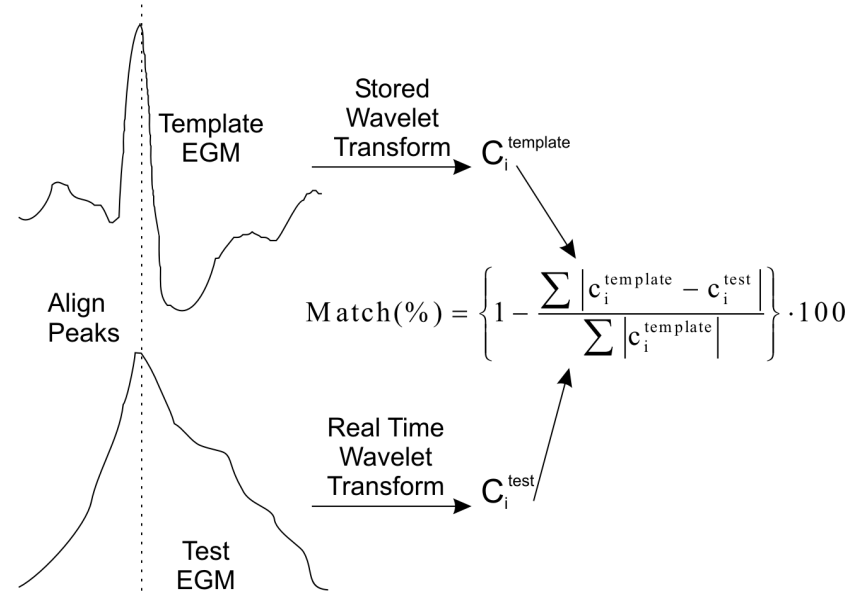

Figure 2. Simplified Wavelet based match score calculation

\section{FREQUENCY DOMAIN}

This algorithm is based on the alterations observed in the spectrum of the complexes (frequency domain) due to morphologic changes between ventricular and supraventricular rhythms.

When the rhythm is originated in the ventricle, the high frequency components of the complex spectrum are attenuated, in opposition to supra-ventricular rhythms, where the spectrum contains high frequency components.

For SVT rhythms, the frequency spectrum has their maximum around $8 \mathrm{~Hz}$ and decrease slowly (figure 3). In the case of ventricular rhythms, the spectrum is characterized by a main frequency peak, typically around $4 \mathrm{~Hz}$ (figure 4).

The algorithm implementation steps are as follows:

1. Current rhythm one complex extraction.

2. Complex spectrum computation using FFT. Only five spectral components are computed and normalized at 4, 8, 12, 16 and $20 \mathrm{~Hz}$.

3. Complex classification using a neural network. When the neural network output is close to 0 , the rhythm is classified as SVT. Otherwise, the rhythm is classified as ventricular. 


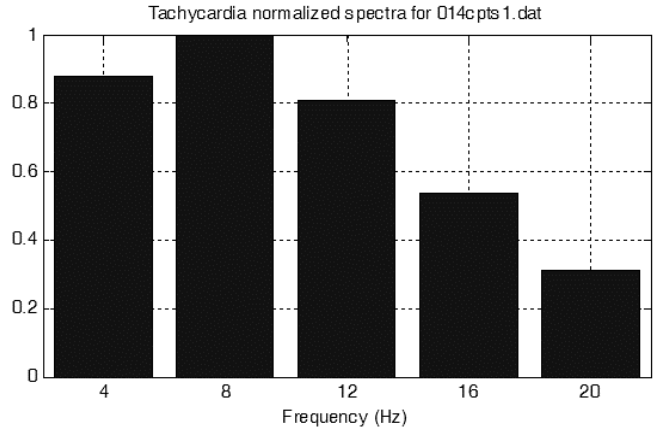

Figure 3. Normalized Fourier spectrum for a SVT complex

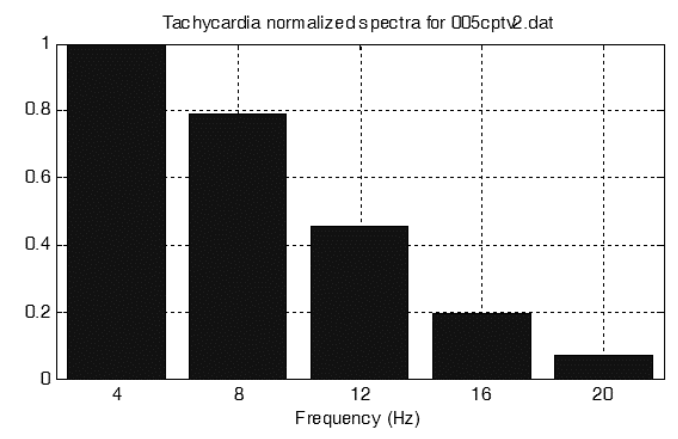

Figure 4. Normalized Fourier spectrum for a VT complex

The neural network (figure 5) has one input layer composed of 5 neurons, a hidden layer of 4 neurons and an output layer of 1 neuron. The original implementation uses an output layer of 2 neurons but, for comparison reasons, the discrimination between VT and VF has been removed. The neural network has been defined and trained using MATLAB Neural Network Toolbox.

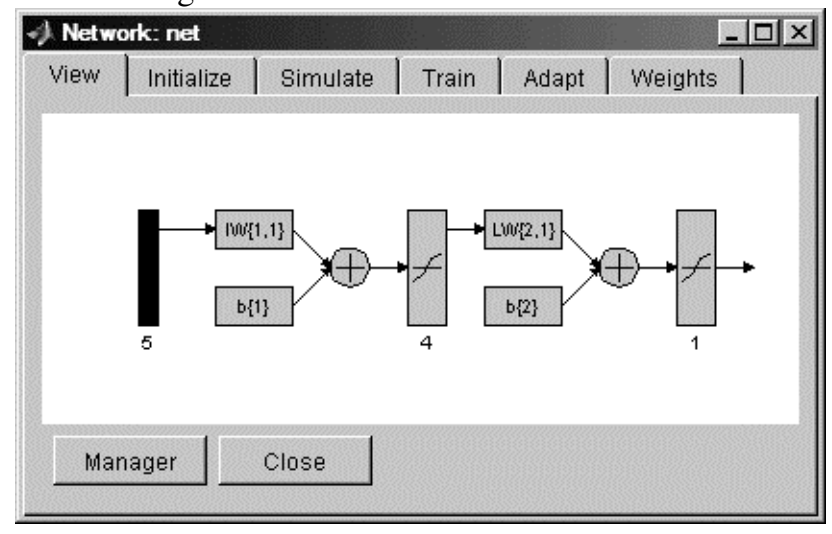

Figure 5. Complex classification neural network

\section{Materials}

An own classified EGM episode database including ICD stored episodes and electrophysiology study episodes has been used. This database includes both bipolar and unipolar recordings.

For the time domain algorithm, the test set includes 52 TV and 29 SVT bipolar EGM episodes.

For the simplified Wavelet algorithm and the frequency domain algorithm the test includes $29 \mathrm{TV}$ and 17 SVT unipolar EGM episodes.

\section{Methods}

All three selected morphological algorithms have been implemented in MATLAB for test and optimization.

The algorithm performance is quantified by sensitivity (SE), specificity (SP) and accuracy (AC). In order to find the optimal settings of the algorithms, different parameter values were evaluated, by varying them within a wide range. The optimization result shows the best combination values for the parameters.

The optimized algorithm is then implemented in a hardware platform based on a 16-bit microcontroller for final test. The hardware platform uses a Texas Instruments MSP430 family microcontroller [7] and has been programmed using $C$ language.

The final test is needed because of differences between MATLAB and C algorithm implementation. MATLAB algorithm implementation works offline and uses float point arithmetic but $\mathrm{C}$ algorithm implementation works in real-time and uses fixed point arithmetic.

\section{Results}

The results obtained from the testing and optimizing process over the three selected morphological discrimination algorithms are:

\section{TIME DOMAIN}

In the optimization process two variables have been included: the percent match score threshold (5 to $95 \%$ range in 5\% steps) and the number of complexes that exceed the threshold in an eight complex window (from 4 to 6 , out of 8 complexes).

Selecting the algorithm configurable parameter values that maximize AC, SE and SP:

\begin{tabular}{|c|c||c|c|c|}
\hline Thereshold & Complex & SE (\%) & SP (\%) & AC (\%) \\
\hline \hline $75 \%$ & $4 / 8$ & 86.54 & 62.07 & 77.78 \\
\hline $95 \%$ & $4 / 8$ & 100.00 & 10.34 & 67.90 \\
\hline $40 \%$ & $4 / 8$ & 63.46 & 75.86 & 67.90 \\
\hline
\end{tabular}

\section{SIMPLIFIED WAVELET}

In the optimization process two variables have been included: the percent match score threshold (5 to $95 \%$ sweep in 5\% steeps) and the number of complexes that 
exceed the threshold in an eight complex window (from 5 to 7 , out of 8 complexes).

Selecting the algorithm configurable parameter values that maximize $\mathrm{AC}, \mathrm{SE}$ and $\mathrm{SP}$ :

\begin{tabular}{|c|c||c|c|c|}
\hline Thereshold & Complex & SE (\%) & SP (\%) & AC (\%) \\
\hline \hline $50 \%$ & $6 / 8$ & 86.21 & 88.24 & 86.96 \\
\hline $75 \%$ & $6 / 8$ & 100.00 & 41.18 & 78.26 \\
\hline $30 \%$ & $7 / 8$ & 58.62 & 100.00 & 73.91 \\
\hline
\end{tabular}

\section{FREQUENCY DOMAIN}

In the optimization process one variable has been included: the number of complexes that match the criterion in an eight complex window (1 to 8 out of 8 complexes).

Selecting the algorithm configurable parameter values that maximize AC, SE and SP:

\begin{tabular}{|c||c|c|c|}
\hline Complex & SE (\%) & SP (\%) & AC (\%) \\
\hline \hline $5 / 8$ & 75.86 & 76.47 & 76.09 \\
\hline $8 / 8$ & 96.55 & 29.41 & 71.74 \\
\hline $1 / 8$ & 37.93 & 100.00 & 60.87 \\
\hline
\end{tabular}

The $\mathrm{C}$ language algorithm implementation for the hardware platform based in a 16-bit microcontroller running at a clock frequency of $32,768 \mathrm{~Hz}$, reveals an increasing complexity in algorithms:

\begin{tabular}{|l|c|c|}
\hline Algorithm & $\begin{array}{c}\text { Execution } \\
\text { cycles }\end{array}$ & $\begin{array}{c}\text { Response } \\
\text { Time }\end{array}$ \\
\hline \hline Time domain & 49,976 & $1.53 \mathrm{~s}$ \\
\hline Simplified Wavelet & 96,032 & $2.93 \mathrm{~s}$ \\
\hline Frequency domain & 287,513 & $8.77 \mathrm{~s}$ \\
\hline
\end{tabular}

\section{Discussion and conclusions}

Performance comparison between algorithms shows that the best results are obtained for the simplified Wavelet, with a percent match score threshold of $50 \%$ and a condition that at least 6 complexes match the criterion. In the case of the frequency domain, the best performance is achieved with a condition that at least 5 complexes match the criterion.
Algorithm complexity was evaluated in terms of execution cycles and response time consumption shows that the frequency domain algorithm has the highest complexity. Assuming a maximum response time of $5 \mathrm{~s}$ for ICD discrimination both time domain and simplified Wavelet algorithms can be implemented in an ICD but frequency domain algorithm cannot.

\section{References}

[1] Swerdlow CD. Supraventricular Tachycardia-Ventricular Tachycardia Discrimination Algorithms in Implantable Cardioverter Defibrillators: State-of-the-Art Review. Journal of Cardiovascular Electrophysiology 2001;12(5):606-12.

[2] Boriani G, Biffi M, Frabetti L, Lattuca JJ, Branzi A. Clinical Evaluation of Morphology Discrimination: an Algorithm for Rhythm Discrimination in Cardioverter Defibrillators. Journal of Pacing and Clinical Electrophysiology 2001;24(6):994-1001.

[3] Swerdlow CD, Brown ML, Lurie K, Zhang J, Wood NM, Olson WH, Gillberg JM. Discrimination of Ventricular Tachycardia from Supraventricular Tachycardia by a Downloaded Wavelet-Transform Morphology Algorithm: A Paradigm for Development of Implantable Cardioverter Defibrillator Detection Algorithms. Journal of Cardiovascular Electrophysiology 2002;13(5):432-441.

[4] Koyrakh LA, Gillberg JM, Wood NM. Wavelet transform based algorithms for EGM morphology discrimination for implantable ICDs. Computers in Cardiology 1999;343-346.

[5] Minami K, Nakajima N, Toyoshima T. Real-Time Discrimination of Ventricular Tachyarrhythmia with Fourier-Transform Neural Network. IEEE Transactions on Biomedical Engineering 1999;46(2):179-185.

[6] Cebrian A, Rey J, Millet J, Rieta JJ, Garcia-Civera R. Comparative Analysis in Terms of Computational Cost for Different Discrimination Algorithms in Implantable Defibrillators. Computers in Cardiology 2004;31:17-20.

[7] Texas Instruments. MSP430x1xx Family User's Guide. 2004.

Address for correspondence

Antonio Cebrian

Universidad Politecnica de Valencia

Dept. de Ingenieria Electronica

Camino de Vera s/n

46022 Valencia (Spain)

acebrian@eln.upv.es 